\title{
Hiponatremia na fase precoce do traumatismo raquimedular
}

\author{
Carlos Umberto Pereira', Julianne Alves Machado² \\ Universidade Federal de Sergipe (UFS), Aracaju, SE, Brasil.
}

\section{RESUMO}

Objetivo: Analisar pacientes com traumatismo raquimedular (TRM) e os níveis séricos de sódio nos 14 primeiros dias pós-trauma, tratamento e prognóstico. Método: Trata-se de um estudo prospectivo, longitudinal e descritivo realizado no Serviço de Neurocirurgia do Hospital de Urgência de Sergipe (HUSE). Resultados: Foi prevalente o sexo masculino (80\%). A idade média foi de 38,4. Quanto mais alto o nível da lesão, maior relação com o surgimento de hiponatremia. Conclusão: Por causa da incidência precoce dessa patologia em pacientes pós-TRM, é importante conscientizar a comunidade médica para a importância do rastreamento dessa patologia, evitando o aumento da morbimortalidade.

\section{PALAVRAS-CHAVE}

Hiponatremia/terapia, traumatismos da medula espinal, sódio, prognóstico.

\begin{abstract}
Hyponatremia in the early stage of spinal cord injury

Objective: Aimed to analyze patients with SCl and serum sodium in 14/1 days post-trauma, treatment and prognosis. Method: This study is a prospective, longitudinal, descriptive realized in Emergency Hospital of Sergipe (HUSE), Department of Neurosurgery. Results: It was prevalent among males (80\%). There is higher incidence in the age group below 40 years, with an average age of 38.4. The level of injury has relationship with the onset of hyponatremia. It was not necessary to impose any treatment. There were no deaths in the first two weeks. Conclusion: Due to the incidence of this disease in patients early after SCl is important to find ways to alert the medical community to the importance of screening for this disease, preventing increased morbidity and mortality.
\end{abstract}

\section{KEYWORDS}

Hyponatremia/therapy, spinal cord injury, sodium, prognosis.

\section{Introdução}

O traumatismo raquimedular (TRM) compreende as lesões dos componentes da coluna vertebral em quaisquer porções: óssea, ligamentar, medular, discal, vascular ou radicular. ${ }^{1}$ A maioria das lesões observada na medula espinhal se deve a eventos secundários que ocorrem de minutos a semanas após a lesão primária induzida pela contusão mecânica. ${ }^{2}$

Estudos sugerem que no conjunto dessas alterações está o surgimento de distúrbios eletrolíticos, em especial a hiponatremia, transtorno comum na fase aguda do TRM. ${ }^{3-6}$ Hiponatremia ocorre nas duas primeiras sema- nas após TRM cervical, ${ }^{4,5}$ porém tem sido registrado um número considerável de casos de hiponatremia entre o segundo e o sétimo dia pós-lesão medular.,

Essa é uma das alterações eletrolíticas mais frequentes na prática hospitalar, sendo na maioria das vezes adquirida na admissão. Pode ser diagnosticada quando a concentração sérica de sódio (natremia) se encontra em níveis inferiores a $135 \mathrm{mmol} / \mathrm{l}^{7}$

Em virtude da grande incidência de alterações no equilíbrio do sódio corporal pós-TRM, desenvolvemos a pesquisa em questão com o objetivo de analisar os níveis séricos de sódio nos primeiros 14 dias pós-TRM e correlacionar com o prognóstico, visando à prevenção e à diminuição da morbimortalidade nesses pacientes.

1 Professor adjunto do Departamento de Medicina da Universidade Federal de Sergipe (UFS) e Neurocirurgião do Hospital de Urgência de Sergipe (HUSE), Aracaju, SE, Brasil.

2 Acadêmica de Medicina da UFS, Aracaju, SE, Brasil. 


\section{Revisão da literatura}

O TRM, definido como uma lesão traumática dos elementos neurais no canal espinhal, é um dos mais devastadores eventos, com significativa morbimortalidade. Resulta em vários graus de déficits sensoriais, motores ou autonômicos. ${ }^{8}$

As principais causas de TRM são: acidentes automobilísticos (36\%-48\%), violência (5\%-29\%), atividades de lazer (7\%-16\%), além de quedas e ferimentos por armas branca e de fogo (17\%-21\%). ${ }^{9}$ Estima-se que existam aproximadamente 2,8 milhões de pacientes com lesão medular no mundo, e esse número vem crescendo em razão do progressivo aumento da violência social e no trânsito. No Brasil, por sua vez, acredita-se que o coeficiente de incidência de lesão medular é de 71 novos casos por milhão de habitantes/ano. ${ }^{9}$ Estudos epidemiológicos estimam que a lesão medular ocorra principalmente no gênero masculino (81\%) com idade entre 16 e 30 anos, atingindo a fase mais produtiva de sua vida; aproximadamente $40 \%$ dos pacientes sofrem tetraplegia e $60 \%$ têm paraplegia. ${ }^{10,11}$

A hiponatremia é uma das mais comuns anormalidades bioquímicas na prática clínica. ${ }^{12-14}$ Caracteriza-se pela diminuição do nível sérico de sódio, sendo um limite comum para o menor intervalo de referência de $135 \mathrm{mmol} / \mathrm{l}^{1,4-7,12,15-18} \mathrm{O}$ gênero feminino antes da menopausa parece ter risco aumentado de desenvolver manifestações neurológicas graves e subsequentes sequelas, em consequência da diminuição dos níveis séricos de sódio. ${ }^{19,20}$

Há um crescente reconhecimento de que a hiponatremia é um marcador de mau prognóstico e de que aumenta potencialmente a morbimortalidade. ${ }^{17,21}$ É um distúrbio normalmente assintomático, mas quando acompanhado por manifestações do sistema nervoso central (SNC), resulta em substancial morbidade. ${ }^{4}$ Existe a necessidade de monitoração dos níveis séricos de sódio no sentido de prevenir possíveis complicações graves pós-TRM. ${ }^{15}$

Além dos déficits diretamente relacionados ao trauma, há eventos secundários acarretados pela lesão medular. Pesquisas sugerem que hiponatremia é um distúrbio eletrolítico decorrente da lesão primária que está comumente presente no TRM. -6,11,22 $^{-6}$

Existem inúmeras potenciais causas para o desenvolvimento de hiponatremia pós-TRM.,23 Alguns estudos acerca do assunto estabelecem uma causa multifatorial. ${ }^{4,5} \mathrm{~A}$ hiponatremia no estágio agudo pós-TRM pode se desenvolver por causa de: 1) diluição das reservas corporais totais de sódio com entrada livre de água; 2) redução das reservas de sódio total do corpo como resultado da perda de sódio anormal; ou 3) interação desses mecanismos devido à integridade das interações autonômicas. É de se observar que, além desses, existem outros fatores etiológicos potenciais que estão provavelmente relacionados ou que podem ser utilizados como diagnósticos diferenciais. ${ }^{4,5}$

Sobre as manifestações clínicas, pode-se afirmar que uma diminuição aguda do sódio, para valores inferiores a $125 \mathrm{mmol} / \mathrm{l}$, determina o aparecimento de náuseas e mal-estar geral. Quando essa diminuição é mais acentuada, para valores entre 115 e $120 \mathrm{mmol} / \mathrm{l}$, surgem manifestações moderadas como cefaleia, letargia e obnubilação. O risco de manifestações graves como convulsões, coma, hérnia cerebral interna ou mesmo óbito é maior quando a natremia apresenta valores inferiores a $110-115 \mathrm{mmol} / \mathrm{l}^{24}$

Níveis mais altos de lesão medular relacionam-se com hipotensão arterial e hiponatremia. O mais significativo preditor de hiponatremia é o tipo de lesão, e não o nível acometido por ela. ${ }^{6}$ Não existe uma causa isolada para a patologia, o que ocorre é a existência de seis maiores causas potenciais: primeiro, o efeito estimulante da hipotensão ortostática sobre a secreção do hormônio antidiurético $(\mathrm{ADH})$ pode ser dissociado do efeito inibitório da hipotonicidade, portanto a água é retida apesar da hiponatremia. Segundo, uma ingestão elevada de líquidos pode influenciar na secreção do $\mathrm{ADH}$ induzindo hiponatremia na fase inicial pós-TRM. Terceiro, pode haver um mecanismo renal de manutenção de água independente do controle do $\mathrm{ADH}$ em pacientes tetraplégicos, que podem combinar um defeito intrarrenal da excreção de água com uma redefinição de homeostase. Quarto, uma excessiva excreção renal de sódio no paciente que sofreu TRM que tem associada lesão traumática cerebral severa ou doença cerebral anterior pode levar à síndrome perdedora de sal (SPS). Quinto, pseudo-hiponatremia pode ocorrer secundariamente a uma aparente redução da concentração sérica de sódio quando o plasma é rico em lipídios ou proteínas. Por último, a perda do controle supraespinhal da inervação renal simpática ou o comprometimento do fluxo sanguíneo renal foram pensados como potenciais causas de deterioração da conservação renal de sódio após TRM. ${ }^{4,5,25}$

A avaliação clínica juntamente com a história e o exame clínico-neurológico são muito importantes para identificar potenciais condições predisponentes e para permitir um plano diagnóstico, avaliação e tratamento adequados. Em resumo, estando a hiponatremia estabelecida e diagnosticada pela medida dos níveis séricos de sódio ( $<135 \mathrm{mmol} / \mathrm{l})$, a história, o exame de imagem e o investigação laboratorial são bons auxiliares diagnósticos. Uma história acurada pode revelar pistas para estabelecer a causa da hiponatremia e identificar rapidamente os sintomas. A investigação baseia-se principalmente na avaliação do estado de hidratação do paciente e na concentração de sódio na urina. A medida da concentração urinária de sódio é muito útil 
em pacientes nos quais o status de volume é difícil de se avaliar. $^{26}$

O exame de rotina de urina ou urinálise e a bioquímica urinária são importantes preditores diagnósticos. A urinálise pode contribuir com a pesquisa da densidade urinária. Devem ser avaliados os níveis de sódio urinário e a osmolaridade urinária. ${ }^{24}$

De acordo com a osmolalidade plasmática, a hiponatremia classifica-se em hipertônica, hipotônica e isotônica. A hiponatremia hipotônica é mais frequente e é comumente dilucional por aumento do efeito do hormônio antidiurético. Ocorre principalmente em pacientes hospitalizados e indica excesso relativo de água no líquido extracelular. Ocorre por depleção de sódio e água (hiponatremia hiposmolar hipovolêmica), por depleção de sódio (hiposmolar normovolêmica) ou por excesso de água e sódio com volume de água maior que de sódio (hiposmolar hipervolêmica). ${ }^{27}$

A hiponatremia hipertônica ocorre por movimentação da água do líquido intra para o extracelular por presença de substâncias osmoticamente ativas, a exemplo de manitol, sorbitol, agentes de contraste e estados de hiperglicemia. ${ }^{27}$

A hiponatremia isotônica ou pseudo-hiponatremia ocorre quando há estados de hiperlipidemia ou hiperproteinemia, fazendo com que a porção aquosa do plasma esteja reduzida. A elevação de lipídios ou proteínas no plasma pode causar diminuição artificial do sódio sérico por causa da maior proporção relativa de volume do plasma. Pode ocorrer também em casos de intervenções cirúrgicas, nas quais o uso de soluções de irrigação livres de sódio faz com que estas cheguem à circulação. ${ }^{27}$

No caso da hiponatremia hiposmolar hipovolêmica, a depleção de sódio e água acarreta diminuição do volume plasmático, que estimula a liberação do hormônio antidiurético, retendo água e desenvolvendo hiponatremia. A perda de sódio, seja ela renal ou extrarrenal, é responsável por esse distúrbio. A SPS está enquadrada nesse grupo e, mesmo sendo controversa, sabe-se que se trata de uma causa potencial de hiponatremia em pacientes com afecções do SNC e pós-neurocirurgia, tendo como diagnóstico diferencial a síndrome de secreção inapropriada de $\mathrm{ADH}$ (SIADH). São estudados dois mecanismos possíveis para sua causa: a alteração do controle neural do rim e a liberação de fatores natriuréticos. $^{28}$

O sistema nervoso simpático está envolvido na reabsorção de sal, água e ácido úrico pelo túbulo contorcido proximal e tem importante papel em regular a liberação de renina. A alteração no controle neural do rim poderia explicar a falha na capacidade de reabsorção de sódio e urato no túbulo contorcido proximal e o comprometimento na liberação de renina e aldosterona. $\mathrm{O}$ segundo mecanismo seria a liberação, pelo cérebro lesado, de fatores que prejudicam a reabsorção de sódio pelos túbulos; e o principal fator responsável parece ser o peptídeo natriurético do tipo $\mathrm{B}^{28}$ Conjuntamente, essas alterações levam ao aumento da excreção urinária de sódio, e essa perda leva à depleção de volume. A hipovolemia estimula os barorreceptores e, com isso, há liberação de hormônio antidiurético, e esse hormônio compromete a capacidade do rim de reabsorção do sal filtrado. Clínica e laboratorialmente, a SPS e a SIADH são similares, mas é importante a diferenciação entre elas, pois, enquanto na primeira há depleção de volume, na última há normovolemia, ou mesmo hipervolemia discreta. $^{24}$

Pesquisas sugerem que as principais causas do desenvolvimento de hiponatremia pós-TRM ou em pacientes neurológicos em geral são a SIADH e a SPS. ${ }^{7,15,23,29-36}$

SIADH e SPS são comumente confundidas e devem ser consideradas como diagnósticos diferenciais, estabelecendo causa e consequentemente tratamento ideal. ${ }^{37,38}$ Há dificuldades em definir critérios diagnósticos, pois os achados laboratoriais de SIADH e SPS são muito semelhantes. Estudo realizado recentemente com pacientes com hemorragia subaracnóidea e hiponatremia demonstrou que em $69 \%$ dos pacientes a causa determinada para hiponatremia foi a SIADH, enquanto em apenas $7 \%$ a causa foi a SPS. Isso dá um indicativo de que casos de hiponatremia relacionados a afecções do SNC podem ter como causa prevalente a SIADH, e nisso reside a importância de identificá-la utilizando a SPS como diagnóstico de exclusão, embora existam poucos casos fielmente diagnosticados. ${ }^{39}$

O diagnóstico de SIADH é dado na presença de hiponatremia, baixa osmolalidade plasmática, quando não há desidratação, produção de urina normal ou diminuída, concentração alta de sódio na urina e osmolaridade alta da urina, depois de ter excluído causas como doenças tireóidea, adrenal, renal, problemas cardíacos ou cirrose. A SIADH caracteriza-se basicamente por osmolalidade plasmática baixa, em presença de elevada concentração de hormônio antidiurético e osmolaridade urinária aumentada. ${ }^{20}$

A SPS é identificada por hiponatremia acompanhada de desidratação, poliúria, baixa osmolalidade plasmática, alta concentração de sódio na urina e normal para o aumento da osmolaridade urinária. Em ambos os casos a função renal normal é um pré-requisito para os diagnósticos. ${ }^{40}$

Com relação ao tratamento, deve-se levar em conta a taxa de correção da hiponatremia, pois se sabe que mudanças agudas na concentração de sódio sérico podem causar graves sintomas neurológicos, tais como náuseas, letargia, mal-estar, cefaleia, desorientação, coma ou até mesmo óbito. ${ }^{41}$ Isso ocorre porque a mudança da concentração plasmática causa um movimento de água 
devido ao seu gradiente osmótico e o cérebro sofre uma espécie de confinamento na cavidade craniana (edema). Em caso de hiponatremia desenvolvida em longo prazo, permite que as células cerebrais se adéquem ao novo estado por meio da extrusão de solutos orgânicos, minimizando a diferença de osmolalidade. Dessa forma, uma rápida correção da hiponatremia de início agudo alivia os sintomas, enquanto a correção rápida no caso de hiponatremia crônica gera um movimento de água para fora das células e consequente recolhimento celular, acarretando uma síndrome de desmielinização osmótica. Nesse caso, déficits podem surgir após o tratamento e estes podem se tornar permanentes. ${ }^{42}$

Assim, o tratamento depende principalmente do tempo de instalação do quadro e da causa estabelecida. Embora os valores sejam variáveis entre os autores, deve ser obedecida a regra para taxa de correção da hiponatremia, que deve ser limitada a menos de 10$12 \mathrm{mmol} / \mathrm{l}$ nas primeiras $24 \mathrm{~h}$ e $18 \mathrm{mmol} / \mathrm{l} \mathrm{em} 48 \mathrm{~h} .{ }^{43}$ Mesmo com supervisão, cuidados e taxa de infusão consideravelmente lenta, deve-se reconhecer que o aumento do sódio sérico não pode ser previsto por fórmulas e que a monitorização frequente da produção de urina e a medida da concentração do sódio sérico são obrigatórias. ${ }^{44}$

Nos casos de hiponatremia deplecional a reposição de líquidos com soro fisiológico deve ser instituída. ${ }^{45}$ Para hiponatremia com euvolemia, o tratamento envolve reposição hormonal, se viável, restrição de água e, possivelmente, terapia antagonista para vasopressina com a utilização de fármacos como a demeclociclina. ${ }^{46}$ Pacientes sintomáticos podem precisar da infusão de solução hipertônica salina, que deve ser feita apenas o tempo suficiente para aliviar os sintomas, ou seja, aumentar o sódio no plasma para níveis seguros (120 a $125 \mathrm{mmol} / \mathrm{l}$ ) ou quando o sódio no plasma aumentou de $18 \mathrm{mmol} / \mathrm{l}$. O tratamento básico da hiponatremia hipervolêmica é restrição de líquidos e de sódio e o uso de diuréticos. No caso de correção de hiponatremia crônica, as taxas de correção devem ser rigidamente controladas por meio de frequentes medições do nível sérico de sódio. Com relação à SIADH, na maioria dos casos, a restrição de água é a base da terapia e deve ser indicada uma ingesta nutricional adequada. Nos casos em que a restrição hídrica não é eficaz ou o paciente não pode aderir ao nível de restrição desejado, podem ser usados fármacos antagonistas da vasopressina como a demeclociclina, e outros como diuréticos podem ser utilizados com cuidado para que não haja hipovolemia. Quando há SPS, deve-se repor a volemia e corrigir a deficiência de sódio. ${ }^{27}$

Novos agentes farmacêuticos estão sendo estudados, como a terapia antagonista do receptor $\mathrm{V} 2$ da vasopressina, com uma classe de agentes conhecidos como vaptans, com eficácia demonstrada inclusive para pacientes com SIADH, porém mais estudos são necessários para comprovação na diminuição da mortalidade. ${ }^{43,47,48}$

Pode-se, então, considerar a hiponatremia como um distúrbio comum na prática clínica e que requer uma gestão cuidadosa. Aqui nos atemos à hiponatremia decorrente de TRM. A hiponatremia é comorbidade relacionada ao período inicial pós-TRM e deve ser cuidadosamente rastreada para que sua causa seja estabelecida, assim o correto tratamento pode ser instituído.

Os adultos jovens do sexo masculino estão predispostos a sofrer TRM, porém os idosos possuem predisposição à hiponatremia, apresentando maior incidência nessa faixa etária, assim como o gênero feminino antes da menopausa tende a apresentar sintomas neurológicos. ${ }^{21,49}$

Sabe-se que a hiponatremia, acompanhada por manifestações advindas do SNC, está relacionada à substancial morbimortalidade. A hiponatremia, no entanto, é assintomática e, na maioria das vezes, benigna e de fácil correção. ${ }^{50-52}$

A correção inadequada da hiponatremia, por sua vez, pode também causar danos ao SNC potencialmente irreversíveis. ${ }^{43} \mathrm{O}$ diagnóstico diferencial entre a SIADH e a SPS é essencial para o tratamento correto do paciente com hiponatremia, prevenindo, assim, maiores danos ou o surgimento de lesão cerebral. ${ }^{52}$

\section{Pacientes e método}

O presente estudo é prospectivo, longitudinal e descritivo. Durante o período de julho de 2011 a julho de 2012, foram coletados dados provenientes da análise dos prontuários dos pacientes acometidos por TRM, de ambos os gêneros e sem restrição de idade, internados no serviço de neurocirurgia do HUSE (Aracaju, SE).

Foram analisados dados epidemiológicos, exames de neuroimagem e níveis séricos de sódio nos 14 primeiros dias pós-trauma, assim como se avaliaram o prognóstico e o tratamento, quando estabelecido.

\section{Resultados}

Foram coletados dados referentes a 70 pacientes, sem restrição de idade e sexo, diagnosticados com TRM nos primeiros 14 dias pós-trauma. As idades variaram entre 18 e 70 anos, com média de 38,4 anos. Quanto ao gênero, 56 pacientes (80\%) eram do sexo masculino e $14(20 \%)$, do feminino. Dos 70 pacientes que sofreram TRM, 40 (57\%) estavam em uma faixa etária abaixo dos 40 anos, com média de idade de 27,6 anos. 


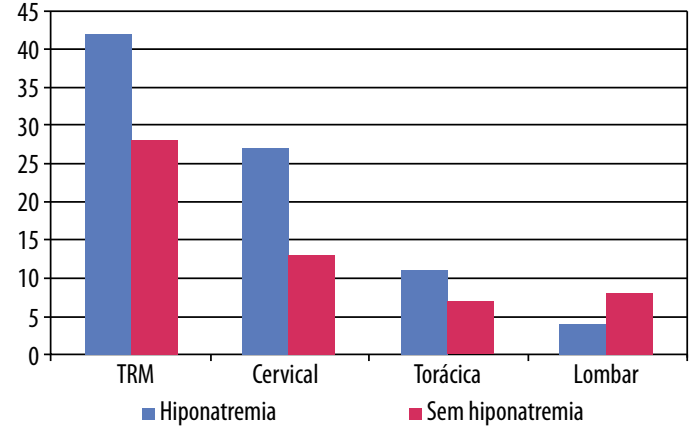

Figura 1 - Prevalência de casos de hiponatremia em pacientes que sofreram TRM. Hiponatremia em casos de lesão cervical, torácica e lombar.

Quanto à presença de hiponatremia nos primeiros 14 dias, 42 pacientes (60\%) desenvolveram hiponatremia e 28 (40\%) não desenvolveram. Os valores dos níveis séricos de sódio nos pacientes que apresentaram hiponatremia variaram entre 128 e $135 \mathrm{mmol} / \mathrm{l}$, com média de $133 \mathrm{mmol} / \mathrm{l}$. Dos 42 pacientes com hiponatremia, 32 pacientes $(76,2 \%)$ apresentaram níveis séricos de sódio entre 130 e $135 \mathrm{mmol} / \mathrm{l}$ e 10 pacientes $(23,8 \%)$ apresentaram níveis inferiores a $130 \mathrm{mmol} / \mathrm{l}$.

A TC foi o exame de escolha para o diagnóstico de TRM. Quanto ao nível da lesão, 40 pacientes $(57,1 \%)$ apresentaram lesão em nível cervical, 18 pacientes $(25,7 \%)$ na coluna torácica e $12(17,2 \%)$ apresentaram lesão em nível lombar (Figura 1). Correlacionando o nível da lesão e o surgimento de hiponatremia, encontramos 63,3\% dos pacientes com lesão em nível cervical, $26,6 \%$ torácico e $10,1 \%$ em nível lombar.

Quanto à época de surgimento da hiponatremia, dentro dos primeiros 14 dias pós-trauma, observamos que, dos 42 pacientes que desenvolveram hiponatremia, $32(76,2 \%)$ desenvolveram até o quinto dia pós-lesão e $10(23,8 \%)$ entre o oitavo e o décimo quarto dia. Vale ressaltar que 23 desses pacientes $(54,8 \%)$ apresentaram hiponatremia nos dois primeiros dias pós-TRM, com média de $133 \mathrm{mmol} / \mathrm{l}$ de sódio.

\section{Discussão}

A hiponatremia é um distúrbio comum na fase aguda pós-TRM nas duas primeiras semanas pós-injúria, com alta prevalência nos dois primeiros dias e maior incidência até o quinto dia. Assim, é confirmada a relação temporal existente entre TRM e o tempo de surgimento de hiponatremia. ${ }^{3-6}$

A literatura médica refere que o surgimento de manifestações clínicas na hiponatremia decorre de uma diminuição aguda da natremia para valores inferiores a $125 \mathrm{mmol} / \mathrm{l}$, estando o risco de manifestações graves associado ainda a maiores reduções nos níveis séricos de sódio. Em nossos pacientes, em razão da pequena alteração na variação dos níveis séricos de sódio, não houve prevalência de sintomas perceptíveis e que pudessem ser relacionados unicamente à hiponatremia.

Neste estudo houve correlação com outros autores que relataram a hiponatremia como sendo na maioria das vezes assintomática e benigna. ${ }^{29,50-52}$ Há concordância no reconhecimento de que a hiponatremia é um marcador de mau prognóstico e que aumenta potencialmente a morbimortalidade quando acompanhada por manifestações do SNC. ${ }^{17}$

Devido à ausência de estado clínico patognomônico para hiponatremia, não houve instituição de qualquer tipo de tratamento específico para hiponatremia, assim como não houve grande preocupação com o rastreamento para essa patologia no local em que foi feito esse estudo. Houve correlação entre os níveis da lesão medular e o surgimento da hiponatremia, sendo o nível alto da lesão medular um preditor de hiponatremia Nenhum paciente em análise evoluiu para óbito nos primeiros 14 dias. Porém, esses pacientes ficarão paraplégicos ou tetraplégicos, afetando a fase mais produtiva de suas vidas. Esse fato ocorre por causa do aumento constante da violência social e no trânsito. Por serem problemas frequentes no cotidiano social, a atenção a essa patologia deve ser enfatizada quando o paciente é admitido no hospital com história de TRM.

\section{Conclusões}

Neste estudo, o sexo masculino foi mais acometido, com maior incidência na faixa etária inferior a 40 anos. O nível da lesão medular é preditor de hiponatremia. Quanto mais alto o nível da lesão, maior a relação com o surgimento de hiponatremia. Há também uma relação temporal entre o TRM e o desenvolvimento de hiponatremia, dentro dos primeiros 14 dias; em análise houve maior prevalência até o quinto dia pós-injúria. Não houve necessidade de se instituir tratamento específico e não houve óbito nas duas primeiras semanas em nossos pacientes. A partir do conhecimento da incidência de hiponatremia pós-TRM e sua prevalência nas duas primeiras semanas pós-lesão, deve-se buscar formas de alertar a comunidade médica para a importância do rastreamento dessa patologia, evitando-se o aumento da morbimortalidade.

\section{Agradecimento}

Este trabalho foi realizado com o auxílio financeiro da Bolsa de Iniciação Científica do Programa Institu- 
cional de Bolsas de Iniciação Científica da Coordenação de Pesquisa da Universidade Federal de Sergipe (PIBIC/ COPES/UFS).

\section{Conflito de interesses}

Os autores declaram inexistência de conflito de interesses na realização deste trabalho.

\section{Referências}

1. Suhardjono. Hyponatremia, prevalence, diagnosis, and management. Acta Med Indones. 2011;43(3):149-51.

2. Del Bel EA, Silva CA, Mladinic M. O trauma raquimedular. Coluna/Columna. 2009;8:441-9.

3. Biyani A, Inman CG, el Masry WS. Hyponatraemia after acute spinal injury. Injury. 1993;24(10):671-3.

4. Furlan JC, Fehlings MG. Hyponatremia in the acute stage after traumatic cervical spinal cord injury: clinical and neuroanatomic evidence for autonomic dysfunction. Spine (Phila Pa 1976). 2009;34(5):501-11.

5. Furlan JC, Krassioukov AV, Fehlings MG. Hematologic abnormalities within the first week after acute isolated traumatic cervical spinal cord injury: a case-control cohort study. Spine (Phila Pa 1976). 2006;31(23):2674-83.

6. Peruzzi WT, Shapiro BA, Meyer PR Jr, Krumlovsky F, Seo BW. Hyponatremia in acute spinal cord injury. Crit Care Med. 1994;22(2):252-8.

7. Tisdall M, Crocker M, Watkiss J, Smith M. Disturbances of sodium in critically ill adult neurologic patients: a clinical review. J Neurosurg Anesthesiol. 2006;18(1):57-63.

8. Massod Z, Wardug GM, Ashraf J. Spinal Injuries: experience of a local neurosurgical centre. Pak J Med Sci. 2008;24:36871.

9. Masini M. Estimativa da incidência e prevalência de lesão medular no Brasil. J Bras Neurocirurg. 2001;12:97-100.

10. Defino HLA. Trauma raquimedular. Medicina Ribeirão Preto. 1999;32:388-400.

11. Harrop JS, Sharan AD, Vaccaro AR. The case of neurologic deterioration after acute cervical spinal cord injury. Spine (Phila Pa 1976). 2001;26(4):340-6.

12. Asadollahi K, Beeching N, Gill G. Hyponatraemia as a risk factor for hospital mortality. QJM. 2006;99:877-80.

13. Moritz ML, Ayus JC. Preventing neurological complications from dysnatremias in children. Pediatr Nephrol. 2005;20(12):1687-700.

14. Paiva WS, Bezerra DA, Amorim RL, Figueiredo EG, Tavares WM, De Andrade AF, et al. Serum sodium disorders in patients with traumatic brain injury. Ther Clin Risk Manag. 2011;7:345-9.

15. Amini A, Schmidt MH. Syndrome of inappropriate secretion of antidiuretic hormone and hyponatremia after spinal surgery. Neurosurg Focus. 2004;16(4):E10.

16. Biswas M, Davies JS. Hyponatraemia in clinical practice. Postgrad Med J. 2007;83(980):373-8.

17. Goh KP. Management of hyponatremia. Am Fam Physician. 2004;69(10):2387-94.
18. Ruíz LFG, Martínez APM. Actualidades en el estudio y manejo de la hiponatremia. Med Int Mex. 2007;23:138-50.

19. Albanese A, Hindmarsh P, Stanhope R. Management of hyponatraemia in patients with acute cerebral insults. Arch Dis Child. 2001;85(3):246-51.

20. Raftopoulos H. Diagnosis and management of hyponatremia in cancer patients. Support Care Cancer. 2007;15(12):1341-7.

21. Tolias CM. Severe hyponatraemia in elderly patients: cause for concern. Ann R Coll Surg Engl. 1995;77(5):346-8.

22. James SH, Ashwini DS, Alexander RV, Gregore JP. The cause of neurologic deterioration after acute cervical spinal cord injury. Spine (Phila Pa 1976). 2001;26(4):340-6.

23. Kageyama K, Suda T. A case of hyponatremia after cervical spinal cord injury. Endocr J. 2011;58(5):369-72.

24. Carvalho CB, Carvalho EPB, Santos VM. Fisiopatologia dos distúrbios do sódio. Brasília Med. 2007;44(1):40-52.

25. Yeates KE, Singer M, Morton AR. Salt and water: a simple approach to hyponatremia. CMAJ. 2004;170(3):365-9.

26. Reynolds RM, Padfield PL, Seckl JR. Disorders of sodium balance. BMJ. 2006;332(7543):702-5.

27. Chubb SA. Hyponatremia treatment guidelines 2007: expert panel recommendations. Clin Biochem Rev. 2009;30(1):35-8.

28. Costa KN, Nakamura HM, Cruz LR, Miranda LS, SantosNeto RC, Cosme SL, et al. Hyponatremia and brain injury: absence of alterations of serum brain natriuretic peptide and vasopressin. Arq Neuropsiquiatr. 2009;67(4):1037-44.

29. Arieff Al. Management of hyponatraemia. BMJ. 1993;307(6899):305-8.

30. Berger TM, Kistler W, Berendes E, Raufhake C, Walter M. Hyponatremia in a pediatric stroke patient: syndrome of inappropriate antidiuretic hormone secretion or cerebral salt wasting? Crit Care Med. 2002;30(4):792-5.

31. Cole CD, Gottfried ON, Liu JK, Couldwell WT. Hyponatremia in the neurosurgical patient: diagnosis and management. Neurosurg Focus. 2004;16(4):E9.

32. Kim DK, Joo KW. Hyponatremia in patients with neurologic disorders. Electrolyte Blood Press. 2009;7(2):51-7.

33. Lath R. Hiponatremia in neurological diseases in ICU. Indian J Crit Care Med. 2005;9:47-51.

34. Mobini B, Ameri E, Behtash $\mathrm{H}$. The prevalence and risk factors of the Syndrome of Inappropriate Anti-Diuretic Hormone Secretion (SIADH) following spinal surgery. J Res Med Sci. 2008;13(3):115-20.

35. Palmer BF. Hyponatraemia in a neurosurgical patient: syndrome of inappropriate antidiuretic hormone secretion versus cerebral salt wasting. Nephrol Dial Transplant. 2000;15(2):262-8.

36. Rahman M, Friedman WA. Hyponatremia in neurosurgical patients: clinical guidelines development. Neurosurgery. 2009;65(5):925-35.

37. Brimioulle S, Orellana-Jimenez C, Aminian A, Vincent JL. Hyponatremia in neurological patients: cerebral salt wasting versus inappropriate antidiuretic hormone secretion. Intensive Care Med. 2008;34(1):125-31.

38. Schrier RW, Bansal S. Diagnosis and management of hyponatremia in acute illness. Curr Opin Crit Care. 2008;14(6):627-34.

39. Huda MS, Boyd A, Skagen K, Wile D, van Heyningen C, Watson I, et al. Investigation and management of severe hyponatraemia in a hospital setting. Postgrad Med J. 2006;82(965):216-9.

40. Jayakumar I, Ranjit S, Balasubramaniam C. Hyponatremia in acute neurological disorders - Is always due to SIADH? J Pediatr Nerosci. 2006;1(1):10-5.

41. Bhaskar E, Kumar B, Ramalakshmi S. Evaluation of a protocol for hypertonic saline administration in acute 
euvolemic symptomatic hyponatremia: a prospective observational trial. Indian J Crit Care Med. 2010;14(4):170-4.

42. Widdess-Walsh P, Sabharwal V, Demirjian S, DeGeorgia M. Neurologic effects of hyponatremia and its treatment. Cleve Clin J Med. 2007;74(5):377-83.

43. Vaidya C, Ho W, Freda BJ. Management of hyponatremia: providing treatment and avoiding harm. Cleve Clin J Med. 2010;77(10):715-26.

44. Mohmand HK, Issa D, Ahmad Z, Cappuccio JD, Kouides $\mathrm{RW}$, Sterns RH. Hypertonic saline for hyponatremia: risk of inadvertent overcorrection. Clin J Am Soc Nephrol. 2007;2(6):1110-7.

45. Hew-Butler T, Ayus JC, Kipps C, Maughan RJ, Mettler $S$, Meeuwisse $W H$, et al. Statement of the Second International Exercise-Associated Hyponatremia Consensus Development Conference, New Zealand, 2007. Clin J Sport Med. 2008;18(2):111-21.

46. Garrett RE, Wilhelm S, Maerz G, Bar-Or D. Vasopressin receptor antagonist use in a neurologic rehabilitation center. J Spinal Cord Med. 2010;33(1):65-7.

47. Ghali JK, Farah JO, Daifallah S, Zabalawi HA, Zmily HD. Conivaptan and its role in the treatment of hyponatremia. Drug Des Devel Ther. 2009;3:253-68.
48. Murphy T, Dhar R, Diringer M. Conivaptan bolus dosing for the correction of hyponatremia in the neurointensive care unit. Neurocrit Care. 2009;11(1):14-9.

49. Pérez R, Del Campo SM, Renán S. Aspectos epidemiológicos de la lesión medular de la población del Centro Nacional de Rehabilitación. Rev Mex Med Rehabil. 2008;20:70-82.

50. Al-Salman J, Kemp D, Randall D. Hyponatremia. West J Med. 2002;176(3):173-6.

51. Nzerue CM, Baffoe-Bonnie H, You W, Falana B, Dai S. Predictors of outcome in hospitalized patients with severe hyponatremia. J Natl Med Assoc. 2003;95(5):335-43.

52. Waikar SS, Mount DB, Curhan G. Mortality after hospitalization wilth mild, moderate and severe hyponatremia. Am J Med. 2009;122(9):857-65.

\section{Endereço para correspondência}

Carlos Umberto Pereira

Av. Augusto Maynard, 245/404, São José

49015-380 - Aracaju, SE, Brasil

E-mail: umberto@infonet.com.br 